\title{
Quantitative Susceptibility Mapping to Assess Cerebral Vascular Compliance
}

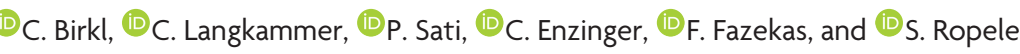
O- $=\nabla \Delta$

\begin{abstract}
SUMMARY: This study explored whether autoregulatory shifts in cerebral blood volume induce susceptibility changes large enough to be depicted by quantitative susceptibility mapping. Eight healthy subjects underwent fast quantitative susceptibility mapping at 3 T while lying down to slowly decrease mean arterial pressure. A linear relationship between mean arterial pressure and susceptibility was observed in cortical and subcortical structures, likely representing vessels involved in autoregulation. The slope of this relationship is assumed to indicate the extent of cerebral vascular compliance.
\end{abstract}

ABBREVIATIONS: $\mathrm{CVC}=$ cerebral vascular compliance; greEPI = gradient-echo echo-planar imaging; MAP = mean arterial blood pressure; QSM = quantitative susceptibility mapping; $\mathrm{SpO}^{2}=$ peripheral capillary oxygen saturation

Q uantitative susceptibility mapping (QSM) is a powerful technique to assess the magnetic susceptibility of tissue. ${ }^{1}$ In brain tissue, this property is dominated by the diamagnetic susceptibility of water, but paramagnetic iron and diamagnetic lipids, proteins, or calcifications may cause regional susceptibility variations. ${ }^{2}$ In contrast, the magnetic susceptibility of blood is dominated by the oxygenation level of hemoglobin, with a more paramagnetic susceptibility in venous vessels and a more diamagnetic susceptibility in arterial vessels. ${ }^{3}$

Cerebral autoregulation ${ }^{4}$ serves to maintain a constant cerebral blood flow over a wide range of mean arterial blood pressure (MAP) levels (50-150 mm Hg) to ensure an adequate supply of glucose and oxygen to the brain. This occurs by vasodilation or vasoconstriction of blood vessels with concomitant changes of intravascular blood volume. ${ }^{5}$ Changes in blood volume per a given change in blood pressure are viewed as indicators of cerebral vascular compliance (CVC), which reflects a main component of

Received July 20, 2018; accepted after revision November 1.

From the Department of Neurology (C.B., C.L., C.E., F.F., S.R.), Medical University of Graz, Graz, Austria; University of British Columbia MRI Research Centre (C.B.), University of British Columbia, Vancouver, British Columbia, Canada; and Translational Neuroradiology Unit (P.S.), Neuroimmunology Branch, National Institute of Neurological Disorders and Stroke, National Institutes of Health, Bethesda, Maryland.

This work was funded by the Austrian Science Fund project Nos. 13001 and 14038.

Please address correspondence to Christoph Birkl, PhD, Medical University of Graz, Department of Neurology, Auenbruggerplatz 22, 8036 Graz, Austria, e-mail: christoph.birkl@medunigraz.at, www.neuroimaging.at

-- Indicates open access to non-subscribers at www.ajnr.org

$\equiv$ Indicates article with supplemental on-line table.

Indicates article with supplemental on-line photos.

http://dx.doi.org/10.3174/ajnr.A5933 vascular function. ${ }^{6,7}$ The capability for CVC varies across the cerebral vasculature, depending on the amount of smoothmuscle cells in the vessel wall, the pericyte density, and, most notably, on the length and diameter of the vessels. ${ }^{8}$ In this regard, the most prominent cerebral autoregulation-induced blood volume changes can be expected to occur in subcortical and pial vessels. ${ }^{8}$

This study explored whether QSM allows depicting and mapping of magnetic susceptibility changes in the brain as a consequence of autoregulatory changes in cerebral blood volume following a drop in blood pressure by lying down. ${ }^{9,10}$

\section{MATERIALS AND METHODS \\ MR Imaging}

Eight healthy volunteers ( 6 men, 2 women) with a mean age of 32 years (age range, 28-49 years) participated in this explorative study, which was approved by the local ethics committee of the Medical University of Graz, Austria (EK 29-623 ex 16/17). MR imaging was performed on a 3T MR imaging system (Magnetom Prisma; Siemens, Erlangen, Germany) using a 64-channel head coil. For QSM, we used a 3D gradient-echo echo-planar imaging (greEPI) sequence ${ }^{11}$ with $\mathrm{TR}=50 \mathrm{~ms}, \mathrm{TE}=30 \mathrm{~ms}$, flip angle $=$ $15^{\circ}, \mathrm{FOV}=250 \mathrm{~mm}$, in-plane resolution $=0.65 \times 0.65 \mathrm{~mm}$, slice thickness $=1.5 \mathrm{~mm}$, EPI factor $=15$, scan time $=57$ seconds. This sequence was started immediately after the volunteers had taken their supine position in the MR imaging scanner. To monitor changes in QSM, we repetitively performed this sequence 8 times, resulting in a total acquisition time of 7 minutes and 44 seconds. Thereafter, a T1-weighted true inversion recovery sequence was performed with $\mathrm{TR}=6000 \mathrm{~ms}, \mathrm{TE}=11 \mathrm{~ms}, \mathrm{TI}=500$ 


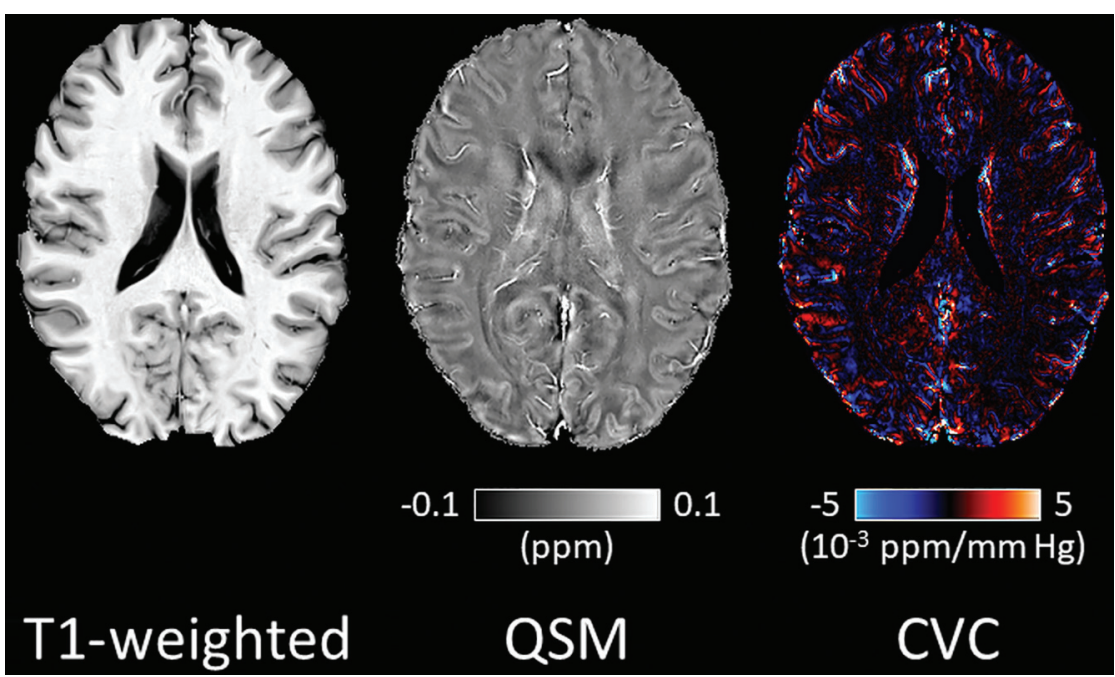

FIG 1. Representative T1-weighted image, quantitative susceptibility map, and the corresponding CVC map of an axial section from a single subject (from left to right). Additional axial sections of the same subject are shown in On-line Fig 4.

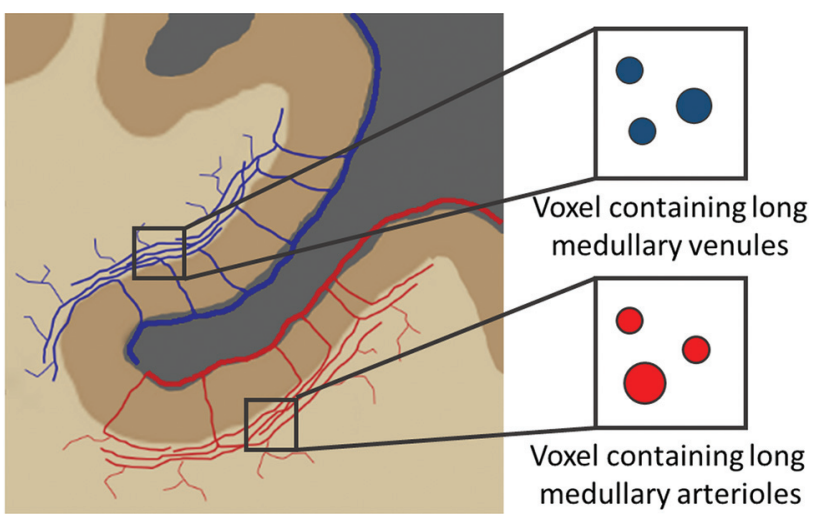

FIG 2. Schematic drawing of pial and subcortical blood vessels. The longest vessels with a high capability to modulate the diameter are the medullary arterioles and venules. ${ }^{16}$ According to Duvernoy type 5 , these vessels follow the course of the subcortical interface over long distances before they branch into white matter. ${ }^{17}$

ms, flip angle $=150^{\circ}$, in-plane resolution $=0.65 \times 0.65 \mathrm{~mm}$, slice thickness $=1.5 \mathrm{~mm}$, acquisition time $=4$ minutes and 44 seconds.

At each start of the greEPI sequence, the systolic (SYS) and diastolic (DIA) blood pressure was measured using a blood pressure cuff at the left upper arm. Furthermore, the blood oxygenation level (peripheral capillary oxygen saturation $\left[\mathrm{SpO}_{2}\right]$ ) was measured at the index finger of the right hand using a MR imaging-compatible patient monitor (Precess; Invivo, Orlando, Florida). The MAP was calculated according to MAP $=D I A+1 / 3(S Y S-D I A) .{ }^{12}$

\section{Image Processing}

QSM images of each greEPI measurement were reconstructed using the total generalized variation method. ${ }^{13}$ To correct for head displacements during the series of greEPI scans, we registered all subsequent scans to the first greEPI scan, using FLIRT (FMRIB Linear Image Registration Tool; https://www.fmrib.ox.ac.uk/fsl/fslwiki/ FLIRT). ${ }^{14}$ Additionally, QSM was referenced to the magnetic susceptibility of the CSF to correct for possible phase drifts. ${ }^{15}$

\section{Assessing Vascular Compliance}

In brain tissue, the magnetic susceptibility of a voxel can be considered as the relative contributions of blood and tissue components. If one assumes normal cerebral autoregulation, a change in blood pressure $(\Delta P)$ introduces a susceptibility shift for a given voxel $\left(\Delta \chi_{\text {voxel }}\right)$ according to

$$
\begin{aligned}
\Delta \chi_{\text {voxel }}(\Delta P)=\Delta V_{\text {blood }}(\Delta P) \times & \\
& \left(\chi_{\text {blood }}-\chi_{\text {tissue }}\right)
\end{aligned}
$$

where $\Delta V_{\text {blood }}$ is the relative change in blood volume and $\Delta P$ the relative change in MAP. A blood pressure decrease results in vasodilation and thus increases the intravascular blood volume (On-line Fig 1). Therefore, voxels containing mainly venous vessels should increase their magnetic susceptibility, whereas voxels containing mainly arterial vessels are expected to show a susceptibility decrease (On-line Fig 2). ${ }^{3}$ This theory is because compared with tissue water, hemoglobin exhibits a negative (diamagnetic) susceptibility when fully oxygenated and a positive (paramagnetic) susceptibility when deoxygenized. ${ }^{3}$ To search for structures exhibiting CVC after the subject lies down, we correlated the magnetic susceptibility of each greEPI measurement with the MAP pixel by pixel using a linear regression model, and we mapped the slope of the linear regression, $\Delta \chi_{\text {voxel }} / \Delta P$, as an indirect measure of CVC.

For quantitative analysis, ROIs were manually defined on the CVC maps in the frontal subcortical white matter of each subject. This region was selected because of the presumed presence of vessels with large CVC according to anatomic studies. ${ }^{16,17}$ The mean slope of all ROIs was taken as indicative of the global CVC of each subject. The sign of the slope was used to distinguish between arterial and venous vessels.

\section{RESULTS}

In every subject, a gradual MAP drop was observed from lying down on the MR imaging table until close to the end of the greEPI scans. The strongest decrease in MAP occurred within the first 4 minutes. The maximal decrease of MAP ranged between 7 and 14 $\mathrm{mm} \mathrm{Hg}$ (mean of the maximum blood pressure change during MRI $=10 \pm 2 \mathrm{~mm} \mathrm{Hg}$ ) across all subjects (On-line Fig 3A). The $\mathrm{SpO}_{2}$ ranged between $96 \%$ and $100 \%$ and was not affected by lying down (On-line Fig 3B).

Figure 1 shows a representative T1-weighted image and corresponding QSM and CVC maps of a single subject. Structures with blood pressure-dependent QSM changes are color-coded depending on the sign of the slope of the pixel-wise regression analysis. These structures are identified primarily on the surface and beneath the cortex. Most likely, they reflect voxels containing predominantly long arterioles (positive slope displayed in red) and venules (negative slope displayed in blue) in the subcortical white 


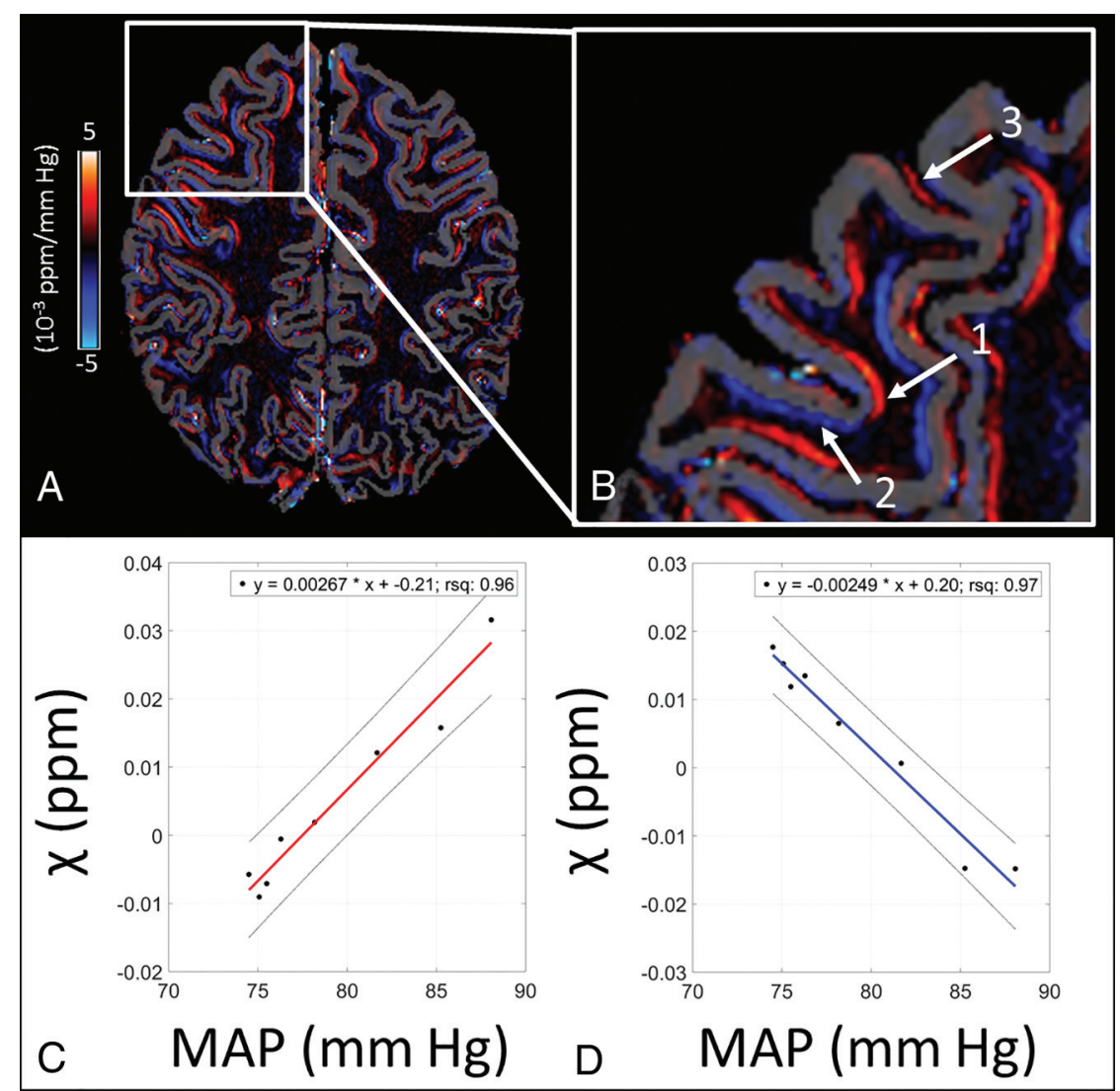

FIG 3. CVC map with a semicortical mask $(A)$ and a zoomed inlay $(B)$ showing regions with mainly arterial (arrow 1), venous (arrow 2), and pial arterial (arrow 3) contributions. In regions with predominantly arterial vessels (arrow 7), the susceptibility increases with increasing MAP $(C)$, while it decreases in regions with predominantly venous vessels (arrow 2) with increasing MAP (D). The slope of the regression lines in $C$ and $D$ represents the $C V C$. rsq indicates the square of the correlation coefficient.

whereby a decrease in blood pressure causes vasodilation and consequently an increase in CBV to keep CBF constant. The slope and direction of the relationship between susceptibility and MAP changes are indicative of the extent of cerebral vascular compliance in respective arterial and venous vessels.

Theoretically, QSM changes could also be caused by a change in the blood oxygenation level. ${ }^{18}$ However, this is unlikely because no physical or mental activity was required during the MR imaging examination. Considering that QSM can also pick up oxygenation-related changes in resting-state fMRI studies, ${ }^{19}$ such changes would be expected to occur predominantly in the cortex and not in such a widespread manner as we have observed. A further argument against an oxygenation-induced origin of our susceptibility changes is the constant $\mathrm{SpO}_{2}$ during the entire MR imaging measurement.

Overall, the proposed approach promises a completely different view of cerebral blood vessels compared with angiographic techniques. Dynamic QSM imaging during changes in blood pressure may serve to assess the functional and not the morphologic component of the cerebral vasculature. Thus, only regions containing vessels with the ability to change their diameter as a consequence of cerebral autoregulation

matter and arterial and venous blood vessels on the brain surface (Duvernoy type 5) $)^{16,17}$ as illustrated in Fig 2.

To illustrate this further, Fig 3 shows a representative compliance map with a semitransparent cortical mask in correspondence with the schematic drawing in Fig 2. The zoomed inlay highlights a subcortical region with mainly arterial contributions (arrow 1), a subcortical region with mainly venous contributions (arrow 2), and a region with pial arterial contributions (arrow 3). The relationship between MAP and susceptibility illustrates that the sign of the regression slope is different for regions with dominating arterial (Fig 3C) and venous (Fig 3D) blood volume changes.

If one assumed a differentiation of arterial and venous blood vessels by the inverse change of susceptibility with decreasing blood pressure, regional evaluation of QSM changes indicated a mean arterial compliance of $2.09 \pm 0.56 \times 10^{-3} \mathrm{ppm} / \mathrm{mm} \mathrm{Hg}$ and a mean venous compliance of $-1.97 \pm 0.56 \times 10^{-3} \mathrm{ppm} / \mathrm{mm} \mathrm{Hg}$ across all subjects (On-line Table).

\section{DISCUSSION}

In this exploratory study, we demonstrate that a small decrease in blood pressure such as that elicited by lying down induces magnetic susceptibility shifts in cortical and subcortical brain structures most likely reflecting contributions from vessels. The direction and extent of susceptibility changes are consistent with cerebral autoregulation, contribute to the calculated CVC maps. ${ }^{20}$ This property appears especially prominent in the long arterioles and venules of the subcortical WM and on the cortical surface. Furthermore, a differentiation between arterial and venous vascular signals is possible on the basis of the different direction of the susceptibility shift of oxygenated and deoxygenated blood. ${ }^{3}$

However, arterial and venous vessels that occupy an imaging voxel in an equal proportion cannot be depicted because their opposite susceptibility shifts will cancel out. The signal from larger vessels with high-flow velocity can also not be captured due to outflow and saturation effects.

The CVC maps represent a relative compliance measure rather than an absolute measure. This feature also explains why the CVC of the venous system seems to be almost as high as the CVC for the arterial system though fewer pericytes are present in the venous system. Apparently, the difference in susceptibility between venous blood and tissue water is much larger $(\sim 1.57 \mathrm{ppm})$ than the difference between arterial blood and tissue water $(\sim 0.26 \mathrm{ppm})$, enhancing the effect of blood volume changes in venous vessels. ${ }^{3}$

\section{CONCLUSIONS}

QSM allows a fast and noninvasive mapping of blood pressureinduced susceptibility changes, which could serve as a measure for CVC. 
Disclosures: Christoph Birkl—RELATED: Grant: Austrian Science Fund, Comments: grant No. I 3001 and J4038.* Christian Langkammer-UNRELATED: Grant: Austrian Science Fund, Comments: Austrian Science Fund grant Nos. KLI523 and P30134.* *Money paid to the institution.

\section{REFERENCES}

1. De Rochefort L, Liu T, Kressler B, et al. Quantitative susceptibility map reconstruction from MR phase data using Bayesian regularization: validation and application to brain imaging. Magn Reson Med 2010;63:194-206 CrossRef Medline

2. Schenck J. The role of magnetic susceptibility in magnetic resonance imaging: MRI magnetic compatibility of the first and second kinds. Med Phys 1996;23:815-50 CrossRef Medline

3. Weisskoff RM, Kiihne S. MRI susceptometry: image-based measurement of absolute susceptibility of MR contrast agents and human blood. Magn Reson Med 1992;24:375-83 CrossRef Medline

4. Bayliss WM. On the local reactions of the arterial wall to changes of internal pressure. J Physiol 1902;28:220-31 CrossRef Medline

5. Lassen NA. Cerebral blood flow and oxygen consumption in man. Physiol Rev 1959;39:183-238 CrossRef Medline

6. Ursino M, Lodi CA. A simple mathematical model of the interaction between intracranial pressure and cerebral hemodynamics. J Appl Physiol 1997;82:1256-69 CrossRef Medline

7. Aaslid R, Newell DW, Stooss R, et al. Assessment of cerebral autoregulation dynamics from simultaneous arterial and venous transcranial Doppler recordings in humans. Stroke 1991;22:1148-54 CrossRef Medline

8. Kontos H, Wei EP, Navari RM, et al. Responses of cerebral arteries and arterioles to acute hypotension and hypertension. Am J Physiol 1978;234:H371-83 CrossRef Medline

9. Panerai R. Assessment of cerebral pressure autoregulation in humans: a review of measurement methods. Physiol Meas 1998;19: 305-38 CrossRef Medline

10. Olufsen MS. Blood pressure and blood flow variation during postural change from sitting to standing: model development and validation. J Appl Physiol 2005;99:1523-37 CrossRef Medline

11. Sati P, George IC, Shea CD, et al. FLAIR ${ }^{\star}$ : a combined MR contrast technique for visualizing white matter lesions and parenchymal veins. Radiology 2012;265:926-32 CrossRef Medline

12. Schmidt RF, Thews G, Lang F, et al, eds. Physiologie Des Menschen. Berlin: Springer-Verlag; 2000 CrossRef

13. Langkammer C, Bredies K, Poser BA, et al. Fast quantitative susceptibility mapping using 3D EPI and total generalized variation. $\mathrm{Neu}$ roimage 2015;111:622-30 CrossRef Medline

14. Jenkinson M, Smith S. A global optimisation method for robust affine registration of brain images. Med Image Anal 2001;5:143-56 CrossRef Medline

15. Liu Z, Spincemaille P, Yao Y, et al. MEDI+0: morphology enabled dipole inversion with automatic uniform cerebrospinal fluid zero reference for quantitative susceptibility mapping. Magn Reson Med 2018;79:2795-803 CrossRef Medline

16. Moody DM, Bell MA, Challa VR. Features of the cerebral vascular pattern that predict vulnerability to perfusion or oxygenation deficiency: an anatomic study. AJNR Am J Neuroradiol 1990;11:431-39 Medline

17. Duvernoy HM, Delon S, Vannson JL. Cortical blood vessels of the human brain. Brain Res Bull 1981;7:519-79 CrossRef Medline

18. Fan AP, Bilgic B, Gagnon L, et al. Quantitative oxygenation venography from MRI phase. Magn Reson Med 2014;72:149-59 CrossRef Medline

19. Sun $H$, Seres $P$, Wilman AH. Structural and functional quantitative susceptibility mapping from standard fMRI studies. NMR Biomed 2017;30 CrossRefMedline

20. Paulson OB, Strandgaard S, Edvinsson L. Cerebral autoregulation. Cerebrovasc Brain Metab Rev 1990;2:161-92 CrossRef Medline 